Original Article

\title{
PROSTHETIC REHABILITATION OF EXTRA-ORAL DEFECTS WITH SILICONE PROSTHESIS
}

\author{
Harshitha Alva ${ }^{1}$, Krishna Prasad D. $^{2}$, Manoj Shetty ${ }^{3}$ \\ ${ }^{1}$ P.G. Student, ${ }^{2}$ Professor and H.O.D., ${ }^{3}$ Professor \\ Department of Prosthodontics and Crown \& Bridge, A.B. Shetty Memorial Institute of Dental Sciences, \\ Nitte University, Deralakatte , M angalore -575018
}

Correspondence:

Krishna Prasad D.

Professor and Head of the Department, Department of Prosthodontics, A.B. Shetty Memorial Institute of Dental Sciences, Nitte University, Deralakatte, Mangalore - 575018 E-mail : drkrishnaprasadd@yahoo.in

\begin{abstract}
:
Man's need for artificial replacements to supply missing or lost body parts has probably existed as long as man himself. Body abnormalities or defects compromise appearance, function and render an individual incapable of leading a relatively normal life. Extraoral defects involving ear, eye, nose, finger and few others are commonly encountered. Prosthetic restoration of these facial defects is an ancient art, in which success has always been limited due to the unavailability of adequate materials or because the available materials do not fulfil the ideal requirements. The most accepted materials to day are the silicones because of their better esthetics.

This paper presents prosthetic rehabilitation of the distal phalynx of left index finger with custom-made silicone prosthesis.
\end{abstract}

Keywords: extra-oral defect, silicone prosthesis

\section{Introduction:}

Prosthetic restoration of facial defects is an ancient art, in which success has always been limited to the availability of materials fulfilling the ideal requirements along with the skill of the specialist concerned. Extra-oral defects involving the ear, eye, nose and finger are routinely seen, amongst which finger amputation is one of the most frequently encountered forms of partial hand loss. Such malfunctions might be due to surgical removal, birth disorders or trauma. Loss is defined by Pertz ${ }^{1}$ as "a state of being deprived of or being without so mething one has had and valued". Patients with such amputations are both physically and psychologically affected. In addition to functional impairment such as grasp strength and security, amputations even affect the socio-economic status'.

\begin{tabular}{|c|}
\hline Access this article online \\
\hline Quick Response Code \\
\hline \\
\end{tabular}

\section{reimplantations have} helped save many severely injured and traumatically amputed digits. However micro vascular reconstruction may be contraindicated, unavailable or unsuccessful in few patients. It is in this group of patients that prosthesis needs to be provided ${ }^{3}$. The prosthesis needs to be as indiscernible as possible, thus restoring the natural appearance, eliminating dysfunction and providing efficient psychological satisfaction. The most preferred material of choice for amputation reconstruction are silicone elastomers.

This paper presents prosthetic rehabilitation of the distal phalynx of left index finger with custom-made silicone prosthesis.

\section{Case Report :}

A 52yr old male patient reported to the department of prosthodontics with a chief complaint of amputed left index finger. History revealed loss of finger during an epileptic seizure 5months back. The amputed finger appeared normal with no signs of infection (figure 1). After a through history and diagnosis, it was decided to fabricate a silicone finger prosthesis.

\section{Procedure:}

A thin layer of petroleum jelly was applied on the patient's 
finger to prevent adherence of the impression material to hair and skin. A wax mould was prepared that would contain the impression material (figure 2). The patient was instructed to keep the hand in normal resting position and impression of the amputed finger was made using irreversible hydrocolloid impression material (Zelgan plusDentsply).

The impression was then poured in type III dental stone and thus a positive replica was obtained. Impression of the index finger of the contra-lateral side was also made and poured with type III dental stone. The model of the other hand served as a guide to develop the contours in the wax pattern for the amputed finger (figure 3).

A wax pattern was then fabricated resembling the contralateral index finger. It was then hollowed from inside by sculpting and placed on the amputed finger cast. Necessary modifications were then made and Try-in was carried out. During try-in, the length and fit of the wax pattern was verified and checked for harmony with the adjacent fingers (figure 4). As the amputed finger itself had a soft tissue bulb like extension, no procedures were carried out for additional retention.

Now the wax pattern along with the cast was invested in a flask with dental plaster and dewaxing was carried out (figure 5). The mould thus obtained facilitates easy packing of the silicone material

The silicone (Cosmesil series materials) was used for this prosthesis. The base colour was dispensed and intrinsic stains were mixed to achieve the desired shade. The dorsal and palmar surfaces were packed separately and characterised individually. The material was then allowed to cure for 24hours. Residual silicone outside the flask was used to check for polymerisation. Once the final prosthesis was retrieved, the flash was trimmed using a sharp blade and final finishing was accomplished using silicone burs. To complete the prosthesis, an artificial nail was fabricated with cold cure clear acrylic resin which was characterised using acrylic stains. The nail was carefully fashioned to match those of the corresponding normal hand. A cynoacrylate adhesive was then applied on the under surface of the nail for bonding with silicone surface to achieve a realistic appearance.

The finger movement was observed to be restricted in the try-in stage. Hence it was decided to shorten the length in the final prosthesis. The final prosthesis was placed on the residual stump and the fit and colour was evaluated (figure 6 and figure 7). The patient was instructed about the maintenance of the prosthesis.

\section{Discussion:}

Loss of even one finger can cause significant functional deficiencies. In addition to immediate loss of grasp strength and security, finger absence also may cause marked psychological trauma. Although the intensity of this trauma varies among individuals, the psychological impact is not related to the magnitude of amputation. Reconstruction of such defects has gained importance in recent years. When surgical restoration is unsuccessful, contraindicated or unavailable, prosthesis definitely provides great psychological help.

Jean Pillet ${ }^{4}$ enumerated the essential characteristics of a prosthesis as the prosthesis should be of high quality both technically and aesthetically, resemble the digit of contralateral hand, skin must correspond to the natural skin in all details and match the colour as appropriately as possible, should not be effected by climatic variations, heat resistant and must not be stained by ordinary materials. Prosthesis must be cleaned easily and should not irritate the skin.

Various materials have been used in the fabrication of these extraoral prosthesis like wood, clay, leather, enamelled porcelain, acrylic resin and silicone elastomers. Over the past decades, silicone elastomers have proven to be the most promising material ${ }^{5}$. Silicones are preferred over other material because of their improved texture, light weight and life like appearance. These silicones are a combination of organic and inorganic compounds. 

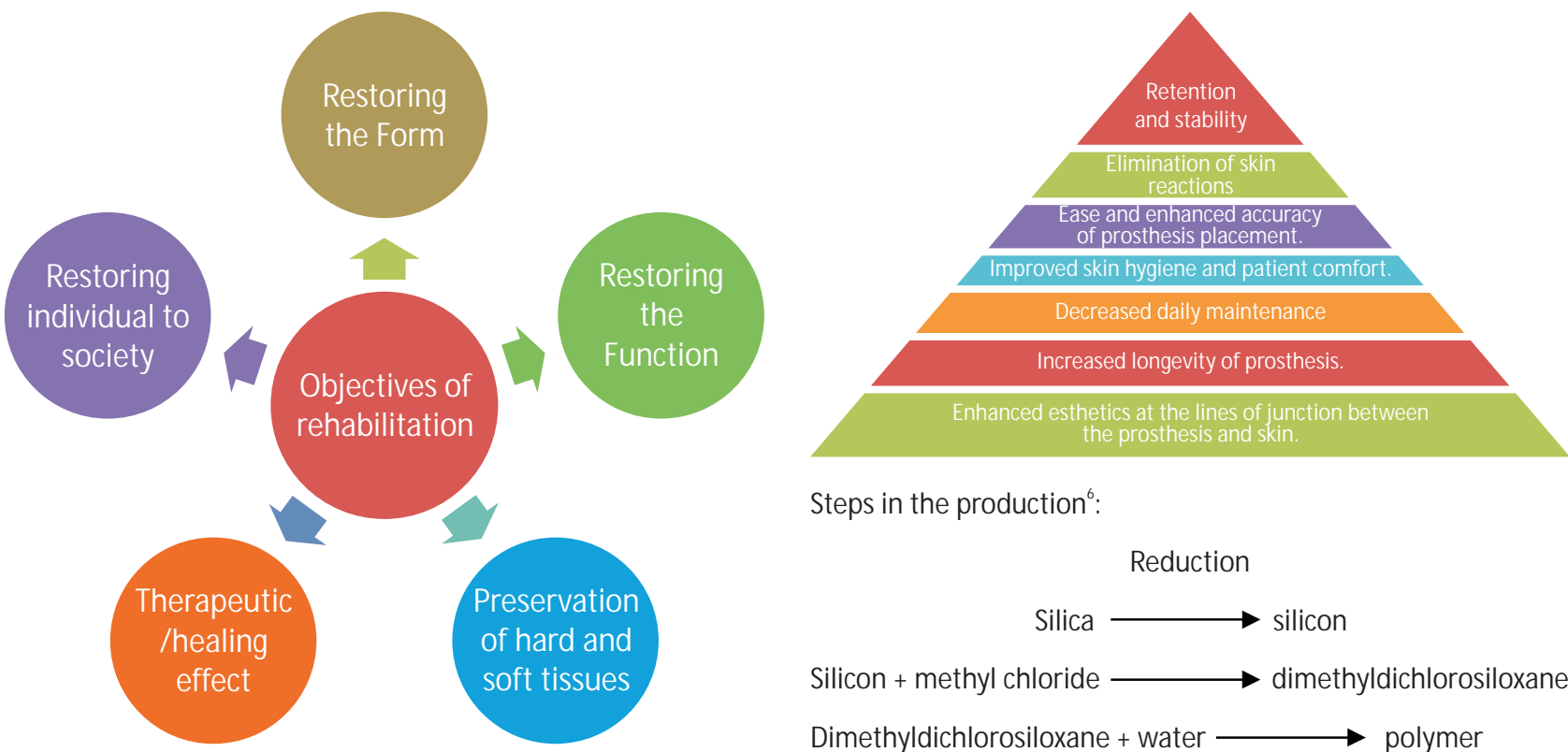

Steps in the production ${ }^{6}$ :

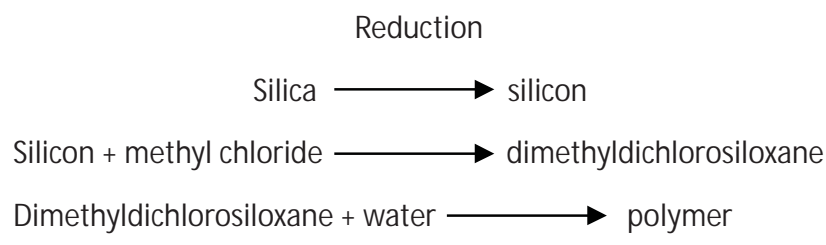

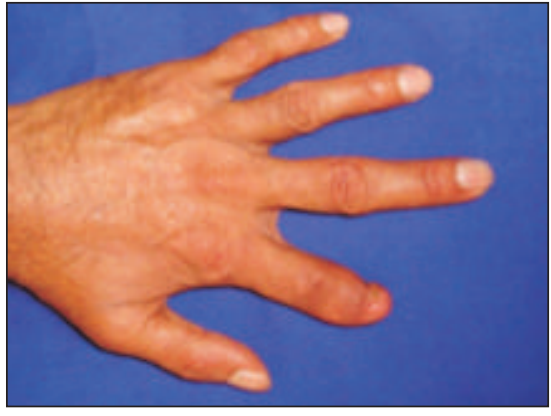

figure 1 : amputed left index finger

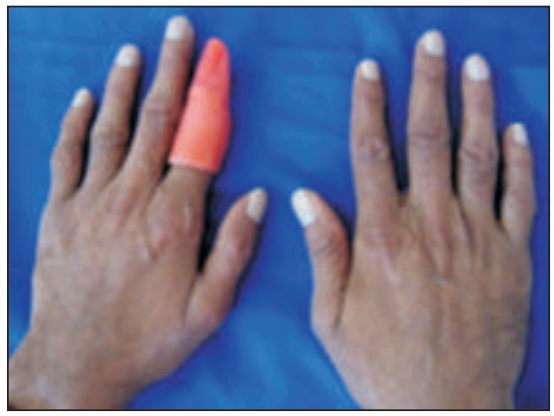

figure 4 : try-in

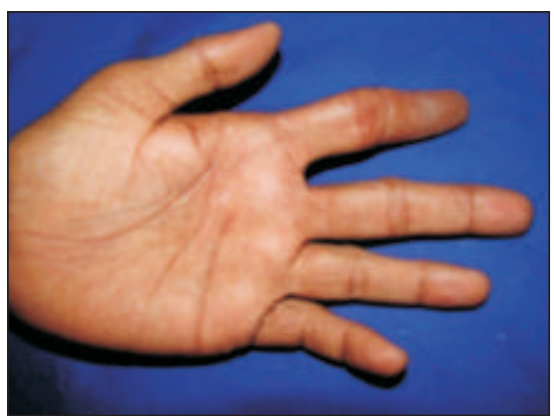

figure 7: final prosthesis (palmar view

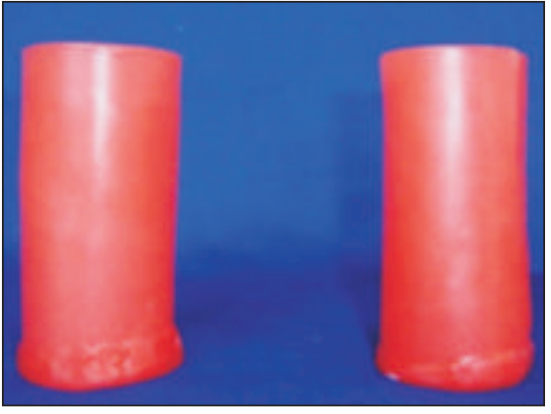

figure 2: wax mould

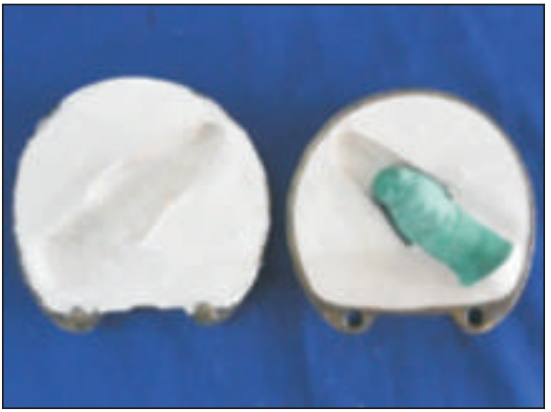

figure 5 : After dewaxing

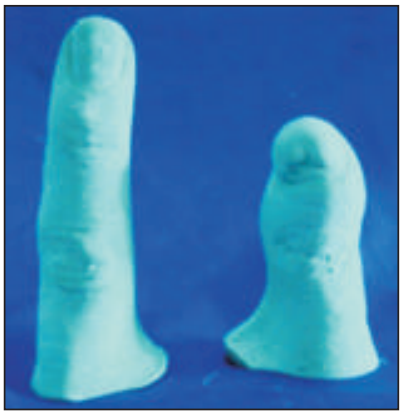

figure 3: cast of index finger of the contra-lateral side and the amputed finger

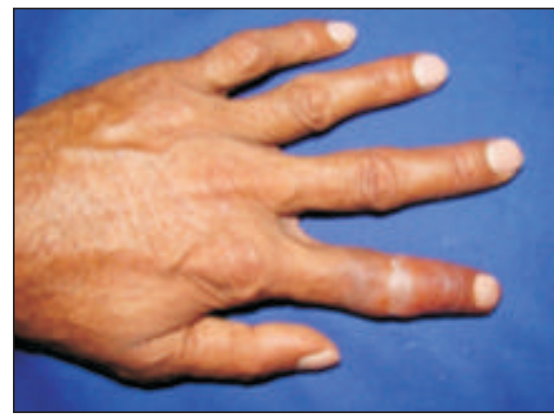

Figure 6 : final prosthesis (dorsal view) To this polymer additives and fillers are added to provide additional strength and colour. Antioxidants and vulcanising agents transform the raw mass from a plastic to a rubbery resin during processing. These silicone elastomers are available in two forms: heat to effect vulcanisation (HTV) and vulcanise at room temperature (RTV). In this case RTV was used in the fabrication of finger prosthesis. 
The durability and resistance to stains such as newsprint, ballpoint ink, clothing dyes, food colouring has made silicone the material of choice these days.

Various methods have been used for the fabrication of wax pattern for the finger prosthesis such as donor technique, or using the impression of the contra-lateral side. JohnJohn ${ }^{7}$ suggested the use of computed tomography data in the affected and non-affected region. With this method the number of visits by the patient can be reduced while replication the finger details immediately and effectively.

Retention is of prime concern and is important for aesthetics, function, and comfort thus enhancing quality. Finger prosthesis is routinely retained by vacuum effect on the stump, use medical grade adhesive or mechanical

\section{References:}

1. Leow ME, Prosthetist C, Pho RW. Optimal circumference reduction of finger models for good prosthetic fit of a thimble-type prosthesis for distal finger amputations.J Rehabil Res Dev. 2001 M ar-Apr;38(2):2739

2. O'Farrell DA, M ontella BJ, Bahor J, Levin LS. Long-term follow-up of 50 Duke silicone prosthetic fingers.J Hand Surg ( $\mathrm{Br}$ Eur) 1996;21B(5):696-700.

3. Aydin C, Karakoca S, Yilmaz H. Implant-retained digital prosthesis with custom-designed attachments: Aclinical report. J Prosthet Dent 2007:97:191-195

4. Shweta D, Saurabh L, Farhan S, Himanshu A. Fabrication of a glove type prosthesis using silicone elastomers.J Indian Prosthodont Soc 2008;8(3):165-168.

5. Aziz T, Waters M, Jagger R. Surface modification of an experimental silicone rubber maxillofacial material to improve wettability. J Dent 2003;31:213-216.

6. Maxillofacial rehabilitation: prosthodontic and surgical considerations. John Beumer.

7. Cabibihan JJ. Patient-specific prosthetic fingers by remote collaboration--a case study. PLoSOne. 2011 M ay 4;6(5):e19508.

8. Livingstone DP. The D-Zstump protector. Am J Occup Ther. 1988 M ar; 42(3):185-7. means such as a finger ring. In the recent years osseointegrated implants have also been used ${ }^{8}$. Silicone elastomers have several advantages over conventional rehabilitave materials.

\section{Conclusion:}

One among the various extra-oral defects is an amputed finger. Finger and partial finger amputations are some of the frequently encountered forms of partial hand loss. Loss of a finger has been found to affect the person psychologically. In such situations restoring aesthetics with sufficient retention becomes the prime concern. Thus a custom-made finger prosthesis using silicone polymers is aesthetically acceptable, partially restores some degree of functionality and comfortable for patient. 